\title{
INFLUENCE OF TWO DIFFERENT IMPLANT DESIGNS ON IMPLANT STABILITY
}

\author{
Maha Nagi *
}

\begin{abstract}
Aim of the study: To evaluate and compare the influence of the conventional solid-structure (CSS) implants versus the direct metal laser sintering (DMLS) implants regarding primary and secondary stability.

Materials and methods: A total of 12 implants of two different designs were tested, six partially edentulous male patients having full set of teeth upper arch opposed by Kennedy class I lower arch with second premolar as last standing abutment bilaterally received twelve implants at the second molar area bilaterally, each patient received two implants of the same size, diameter and implant abutment connection, one conventional solid-structure (CSS) implants (control group) at the right side and one direct metal laser sintering (DMLS) implant (test group) at the left side. Primary and secondary implant stability were measured and compared by resonance frequency analysis using osstell device at time of implant insertion, weeks 1, 4, and 12 after implant insertion.

Result: there was a statistically insignificant difference in stability values between direct metal laser sintering (DMLS) implants (test group) and conventional solid-structure (CSS) implants (control group) throughout the follow up periods except at 12 weeks follow up period there was a statistically significant increase in stability values in direct metal laser sintering (DMLS) implant (test group) compared to the conventional solid-structure (CSS) implants (control group).
\end{abstract}

Conclusion: Direct metal laser sintering (DMLS) implants showed higher secondary stability values than conventional solid-structure (CSS) implants.

KEY WORDS: Implant design, implant stability, osstell device, direct metal laser sintering implants

\section{INTRODUCTION}

The long-term success of oral treatments using implant retained prosthesis is related strongly to the interaction between bone tissue and implant which is called osseointegration. Multiple factors must be considered to obtain successful osseointegration such as patient related factors including systemic condition, oral health, bone quality and quality, as well as the surgical technique used during implant insertion and finally the implant itself including its

* Lecturer, Removable Prosthodontics Department, Faculty of Dentistry, British University in Egypt. 
shape, size, and surface treatment, the relationship between these factors determines implant stability which is classified in two categories: primary stability at time of implant insertion and the secondary stability after the osseointegration period. ${ }^{1,2,3}$

Primary stability is defined as "the absence of movement after their surgical insertion" ${ }^{4,5}$ and is related to the "primary bone contact" between the implant and the prepared bone bed. For this reason, primary stability is mainly related to the surgical site preparation, the bone density and the implant geometry ${ }^{6},{ }^{7}$

The healing process, which follows implant placement, leads to new bone apposition onto the implant surface, thus providing the "secondary stability." When an implant reaches enough secondary stability, it should be ready to successfully support the final prosthetic restoration. The time required to achieve the secondary stability is affected by several variables, such as bone density, implant geometry and surface, surgical technique and finally loading conditions during healing ${ }^{8,9}$. To allow safe functional load, systemic conditions such as smoking, osteoporosis, bisphosphonates assumption and/or diabetes could also influence the implant healing time or loading protocol. ${ }^{10}$

The influence of primary stability on osseointegration depends not only on bone quality and quantity but also on characteristics related to the macroand microgeometry of the screw as diameter, length, shapes, thread pitch, and screw surface roughness. ${ }^{11}$, ${ }^{12}$ The morphological structure characteristics of the screw can function to strengthen the anchoring of the implant to the bone, minimize micromovement and shear forces, minimize crestal bone $\operatorname{loss}^{13}$, promotes proliferation and differentiation of the osteoblast cells, and inhibits fibrous tissue invasion and encapsulation. ${ }^{14,15,16}$

Implant macro and microstructures have been modified over the years to improve both the primary and the secondary stability by maximizing the im- plant surface area in contact with the surrounding bone and better engagement of the marginal cortical bone ${ }^{17}$. An ideal implant macro-geometry should balance the compression and traction forces, minimize generation of shear forces and reduce micromovements to below 50-150 mm during the healing period ${ }^{18},{ }^{19}$.

A tapered implant geometry provides the basis for acceptable primary stability by allowing the gradual expansion of thin crests and minimizing stress at the interface with the surrounding bone ${ }^{20}$.Clinically, it was proven that implants with a tapered design have increased stability in sites with low bone densities or fresh extraction sockets ${ }^{21,22}$. Today, most endosseous dental implants are thread shaped, which provides long-term stability when placed in bone ${ }^{23}$. AbuHammad et al demonstrated that an unthreaded implant design produced lower bone stresses ${ }^{24}$. However, researchers found that a conventional solid-structure implant (CSSI) with a uniform structure could not satisfy many biomechanical requirements, such as biocompatibility, strength, and load bearing ${ }^{25}$.

The narrower implant threads increase the surface area, leading to a more favorable distribution, thereby achieving greater primary stability. Moreover, the tension is more sensitive to the thread's pitch in cancellous bone compared to cortical bone. This observation suggests that the availability of cortical and medullar bone may influence the implant stability ${ }^{18,26}$.

In addition to implant macro-structure role in bone-to-implant contact (osseointegration), a micro-geometric network of interconnected pores is designed for bone ingrowth and propagation throughout the porous structure to augment anchorage of the implant. The combination of bone-toimplant contact and bone ingrowth and propagation within the porous material has been termed osseoincorporation. ${ }^{27},{ }^{28}$

Dental implants currently available nowadays 
in the markets have been produced by machining titanium rods, with subsequent post-fabrication processing and application of surface treatments or coatings, with the aim to promote osseointegration, accelerating the bone healing processes. DLMF is a timesaving metal forming procedure in which a high-power laser beam is directed on a metal powder bed and programmed to fuse particles according to a computer added design (CAD) file, thus generating a thin metal layer. Addition of subsequent layers results in the desired shape of a 3D form with the need of minimal post-processing complications. With Direct metal laser sintering (DLMS), it is now possible to construct dental implants with different shapes and textures, directly from computer added design (CAD) models. ${ }^{29}$

Rapid prototyping or additive manufacturing $(\mathrm{AM})$, is a process to directly generate physical objects with defined structure and shape on the basis of virtual three dimensional (3D) model data. Selective laser sintering, selective laser melting, stereolithography, blown powder cladding, and 3D printing are all common techniques for additive manufacturing. Direct metal laser sintering (DMLS) is an additive metal fabrication technology, utilizes a 3-dimensional CAD model, creating a file which is then forwarded to the software of the direct metal laser sintering machine with a powerful fiber optic laser, within the machine build chamber is a platform for material dispensation and a build platform, in addition to a recoated blade which moves new powder on top of the build platform. Using a focused laser beam, this DMLS technology locally melts metal powder to fuse it together turning it into solid parts. Parts are constructed additively, one layer at a time. Parts built using DMLS have excellent mechanical properties equivalent to that of wrought materials with exceptional resolution, and high surface quality. The metal powder is melted entirely to create a fully dense, homogenous and fine structure. DMLS is a net-shape creates unique geometric freedom of design with cavities and undercuts with great surface quality and excellent mechanical properties. ${ }^{30}$

Titanium and its alloys have been utilized as desirable materials for dental and orthopedic implants because of their excellent corrosion resistance, biocompatibility, mechanical properties, and high strength-to-weight ratio ${ }^{31,32}$. However, one major concern about these titanium implants is the difference between the Young's modulus of bone $(10$ to $30 \mathrm{GPa})$ and titanium $(110 \mathrm{GPa})^{33}$, which leads to insufficiently loaded bone and stress shielded with greater resorption and consequently, implant loosening ${ }^{34}$. Introduction of porosity into titanium and its alloys has been proposed to reduce the Young's modulus of titanium ${ }^{35}$.

Schiefer suggested that the anisotropic behavior of porous titanium must be considered in the construction of implants For these reasons, it is postulated that an unthreaded titanium dental implant with a porous structure would reduce the high stresses at the crestal bone and induce appropriate load sharing between the implant and the surrounding bone. ${ }^{36}$

Primary stability can be assessed before applying the load to predict osseointegration and to select the most adequate type of load for each clinical situation $^{37}$. Currently, insertion torque (IT) and resonance frequency analysis (RFA) are the most commonly used methods for evaluating implant stability. ${ }^{38}$

The objective of this study was to compare the stability of porous implants constructed with direct metal laser sintering (DMLS) with conventional solid-structure (CSSI) implant regarding primary and secondary stability.

\section{MATERIALS AND METHODS}

A total of 12 implants of two different designs were tested. Six partially edentulous male patients having full set of teeth upper arch opposed by Ken- 
nedy class I lower arch with second premolar as last standing abutment bilaterally received twelve implants at the second molar area bilaterally. Each patient received two implants, one conventional solid-structure (CSS) titanium type IV implants at the right side (control group) (Impianto Implus MC straight neck, LEADERS Italia) and one direct metal laser sintering (DMLS) titanium type V implant at the left side (test group) ( Impianto Tixos MC Tixos neck. LEADERS Italia). All implants were $4.1 \mathrm{~mm}$ in diameter and $8 \mathrm{~mm}$ in length with identical implant abutment connection (Morse connection). Figure 1,2

The preoperative examinations included cone beam computed tomography (CBCT) (scaora 3D $X$. Filand) was taken to evaluate and measure the available bone width and height at the lower second molar area

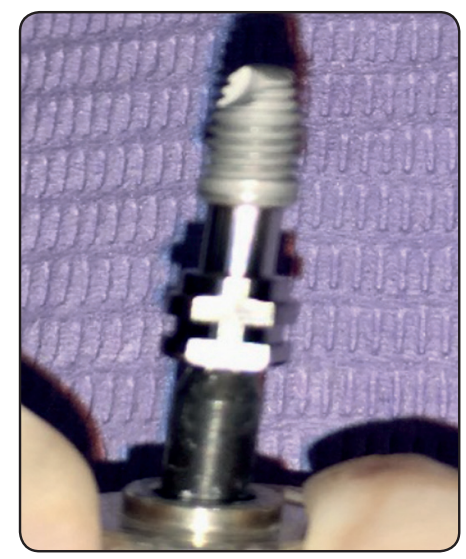

Fig. (1): CSS implants

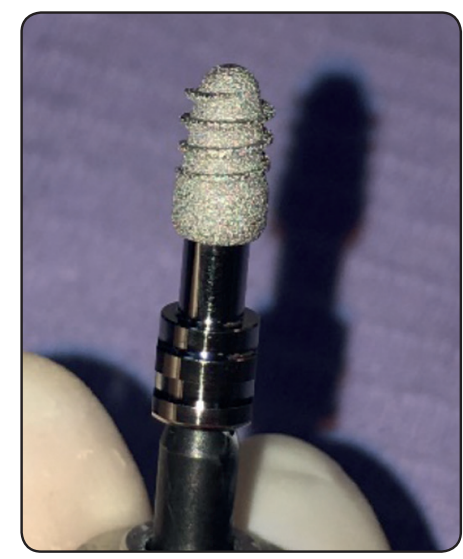

Fig. (2): DMLS implants
Patients had either missing lower posterior molars bilaterally for at least 3 months, or had medical history revealed no contraindication for implant therapy were included in the study, moreover, the existing bone was suitable for implant placement with at least a $4.1 \mathrm{~mm}$ diameter and $8 \mathrm{~mm}$ length, and a minimum bone thickness at the crestal level of $5.1 \mathrm{~mm}$ as examined by CBCT and finally, patient agreed to sign the ICF after the experimental protocol was explained.

However, patients suffering from active infection or inflammation in the areas intended for implant placement, or patients whose general health was poor or had systemic diseases such as diabetes were excluded from the study, moreover, patients with history of radiotherapy for head and neck, patients recieved treatment with bisphosphonates in the last 12 months, and finally patient smokes more than five cigarettes per day were also excluded.

After surgical guide construction, drilling sequence protocol was performed following the manufacturer's recommendation for each type of implant. The drilled sites were irrigated with saline, and bone type and implant insertion torque were recorded during drilling and implant insertion. Bone strength was registered during osteotomy preparation and implant placement based on tactile perception.

Insertion torque values were determined as the maximum torque value $(\mathrm{N} / \mathrm{cm})$ reached at the end of the insertion of the implant in the recipient site.

Primary stability was recorded by resonance frequency analysis (RFA) using an Osstell Mentor device (Ostell/Integration Diagnostics, Goteborg, Sweden). Figure (3) The frequency transducer (SmartPeg) was connected to the implants in a uniform manner perpendicular to the alveolar crest, and the implant stability quotient (ISQ) was measured four times for each inserted screw (buccal, lingual, mesial, and distal) oriented perpendicular to the transducer as recommended 


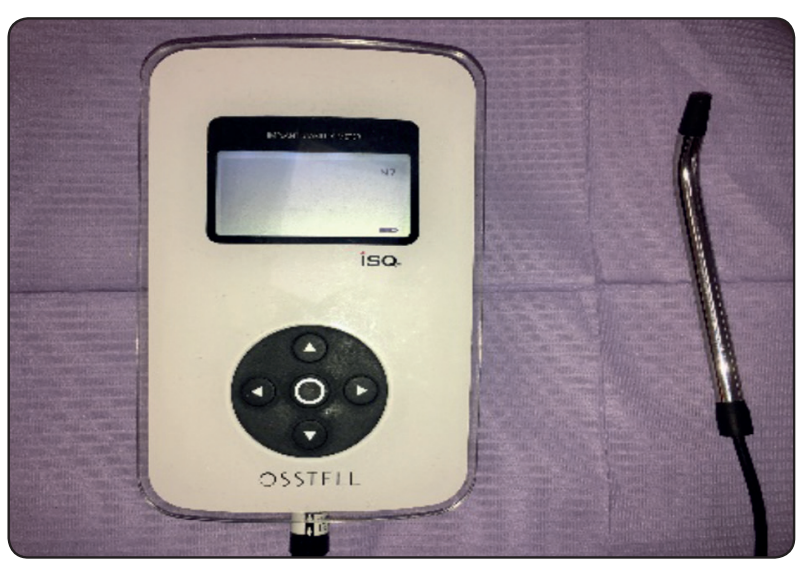

Fig. (3) Osstell device

by the manufacturer. Figure (4) The clinical range of ISQ has been found to be normally at 50 to 80 for implant stability. The secondary stability (SS) was recorded postoperatively at 4,12 weeks. The mean and standard deviation were calculated for subsequent comparison and discussion.

\section{Statistical Analysis}

Statistical analysis was performed using IBM SPSS Statistics Version 2.0 for Windows. Data was presented as mean and standard deviation (SD). The significance level was set at $\mathrm{P} \leq 0.05$. KolmogorovSmirnov and Shapiro-Wilk tests were used to assess data normality.

One-way ANOVA followed by Tukey's post-hoc test was used to compare implant stability quotient at different follow-up periods within conventional solid-structure (CSS) titanium type IV implants and direct metal laser sintering (DMLS) titanium type V implant groups. Independent Student-t test was performed to compare implant stability quotient between CSS and DMLF implant at each follow-up period.

\section{RESULTS}

Comparison of implant stability quotient values (ISQ) between traditional and new implant at each follow-up period:

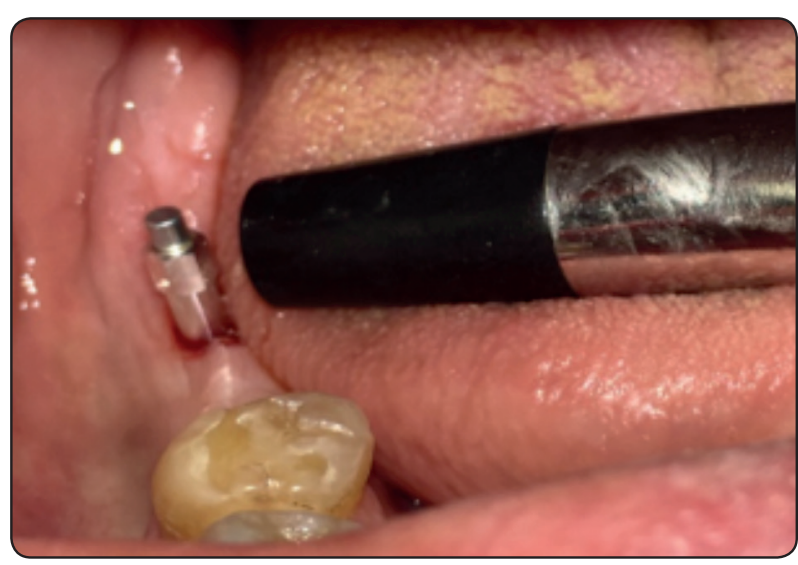

Fig. (4) ISQ measurement

TABLE (1): Mean \pm Standard Deviation (SD) and $\mathrm{P}$ value for the comparison of implant stability quotient values (ISQ) between traditional and new implant at each follow-up period.

\begin{tabular}{|l|l|l|l|}
\hline & CSS implant & DMLF implant & P-value \\
\hline Implant insertion & $58.35 \pm 5 .{ }^{2} \mathrm{~A}$ & $59.45 \pm 3 .{ }^{9} \mathrm{~A}$ & 0.320 \\
\hline 1 week & $57.45 \pm 4 .{ }^{1} \mathrm{~A}$ & $57.03 \pm 3.1 \mathrm{~A}$ & 0.285 \\
\hline 4 weeks & $66.45 \pm 4 .{ }^{0} \mathrm{~A}$ & $73.3 \pm 5 .{ }^{3} \mathrm{~A}$ & 0.152 \\
\hline $\mathbf{1 2}$ weeks & $70.9 \pm 3.8^{\mathrm{B}}$ & $78 \pm 3 .{ }^{4} \mathrm{~B}$ & $0.015^{*}$ \\
\hline P-value & $0.000^{*}$ & $0.000^{*}$ & \\
\hline
\end{tabular}

*: Significant at $P \leq 0.05$ Means with different superscript letters within the same column are significantly different at $P \leq 0.05$

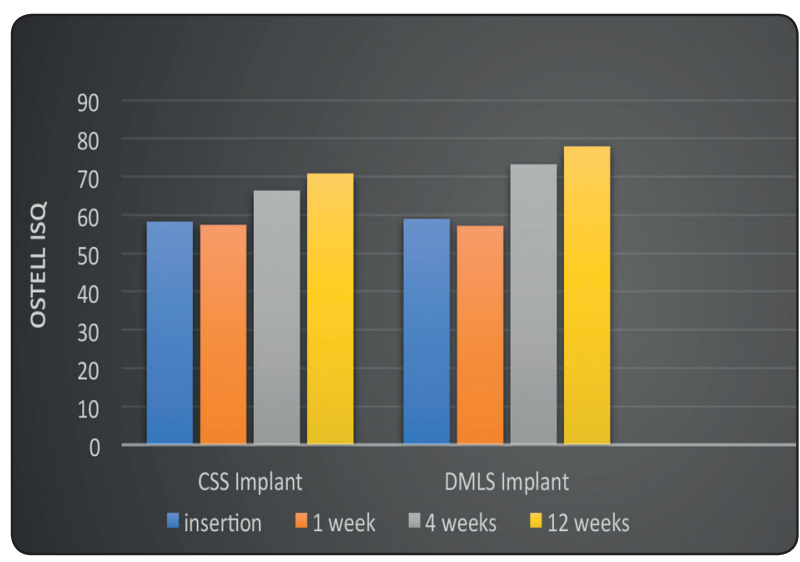

Fig. (5): Comparison of implant stability quotient values (ISQ) between CSS implants and DMLS implants at each follow-up period. 
The two groups showed no statistically significant difference in mean ISQ values at time of implant insertion, one week and four weeks follow up periods as shown in table (1) and figure (5).

At one week follow up period there were a slight decrease in mean ISQ values, followed by increased values throughout the follow up period.

The DMLS implants (test group) showed statistically significant increase in mean ISQ values compared to the CSS implants (control group) at 12 weeks follow up period after implant insertion.

\section{DISCUSSION}

The information obtained from an Osstell -regarding implant stability quotient (ISQ)- can be used to accurately determine stability at the time of placement and later when a decision is being made regarding type of prosthetic restorations.

The present study was done to investigate the effect two different implant designs; the conventional solid-structure (CSS) implants (control group) and the newly designed implant formed by direct metal laser sintering (DMLS) implants (test group) on implant stability in lower second molar area of Kenndey class I partially edentulous patients. The conventional solid-structure (CSS) (control group) implants achieved similar outcomes to the direct metal laser sintering (DMLS) (test group) implants with regarding implant primary stability at implant insertion, one week and 4 weeks follow up periods, the two groups showed statistically insignificant differences of ISQ values. Which could be explained by the presence of implant's threads which increase implant contact area with the bone and improve the load distribution at the implant/bone interface improving primary stability ${ }^{39}$.

According to the manufacturer, both implant designs have the advantages of surface threads, allowing for more rapid installation with less trauma, high cutting power, as many studies indicates that the shape and external characteristics of the screw strongly affect its biomechanics and primary stability ${ }^{40}$.
Adequate stability of an implant is important to allow undisturbed healing and bone formation to occur, thereby permitting optimal stress distribution from masticatory and occlusal functional loads, however, the reduction in ISQ values from time of implant insertion to week one for both groups was noted which were consistent with the observations of previous studies and indicated a declining primary stability. ${ }^{41,42}$ Buser et al speculated that the bone damage resulting from recipient site preparation and ensuing oseteoclast metabolism around an implant fixture might cause reductions in primary stability. ${ }^{43}$ Instead, subsequent increases in ISQ values indicated increases in secondary stability, osseointegration, and overall implant stability. ${ }^{44},{ }^{45}$

The non-significant differences from Week one to Week 4 and the significant difference from Week 4 to Week12 indicated that both groups reached a stability plateaus only at Week 12 . Bone healing or remodelling therefore could persist for 10 to 12 weeks postoperatively. ${ }^{46}$

In another study, a titanium beaded porous surface was applied to cylindric dental implant surfaces previously. Deporter et al ${ }^{47}$ tested implants with sintered bead surfaces placed in canine extraction sockets and reported that the percentage of bone ingrowth inside a sintered bead implant surface was $52 \% \pm 16 \%$ at 4 weeks and exhibited no significant change $(51 \% \pm 16 \%)$ at 8 weeks.

The Direct Metal Laser Sintering technology forms implant morphology with a relatively high porosity at the surface and a high density in the core, which replicates the bone spongy geometry; such a structure - impossible to obtain through the traditional treating surface processes - is highly mimetic, thus accelerating bone healing, enhancing faster osseointegration, enhancing 3D organization of fibrin network, cell adhesion and migration, with exchange of fluids and nutritional elements and the development of good vascularization for proper bone matrix organization. The $3 \mathrm{D}$ geometry created by micro and macro-cavities of well-defined sizes 
and form, interconnected by micro-pores, promotes new bone formation. The concavities, with size 2 to 200 microns, penetrate deep inside the implant body down to 250 microns, creating interconnected pits and pores that are colonized by bone cells ${ }^{29}$, explaining the increase in implant stability at 12 weeks follow up period.

\section{CONCLUSION}

Within the limitation of this study, direct metal laser sintering (DMLS) implants has higher secondary stability values than conventional solid structure (CSS) implants.

\section{REFERENCES}

1. Chang CL, Chen CS, Huang $\mathrm{CH}$ and Hsu ML. Finite element analysis of the dental implant using a topology optimization method. Med Eng Phys. 2012; 34:999-1008.

2. Desai SR, Desai MS, Katti G and Karthikeyan I. Evaluation of design parameters of eight dental implant designs: a two-dimensional finite element analysis. Niger J Clin Pract. 2012; 15: 176-181.

3. Suer BT, Yaman Z, Buyuksarac B. Correlation of fractal dimension values with implant insertion torque and resonance frequency values at implant recipient sites. Int J Oral Maxillofac Implants. 2016; 31: 55-62.

4. Wilson TG Jr, Miller RJ, Trushkowsky R, Dard M. Tapered implants in dentistry: revitalizing concepts with technology: a review. Adv Dent Res. 2016; 28: 4-9.

5. Xu C, Wei Z, Liu N, et al. The effect of implant shape and screw pitch on microdamage in mandibular bone. Clin Implant Dent Relat Res. 2013;17:365-372.

6. Marquezan, M., Osorio, A., Sant'Anna, E., Souza, M.M. and Maia, L. Does bone mineral density influence the primary stability of dental implants? A Systematic Review Clinical Oral Implants Research 2012; 23: 767-774.

7. Gehrke, S.A., da Silva Neto, U.T. and Del Fabro, M. Does implant design affect implant primary stability? A Resonance Frequency Analysisbased randomized split-mouth clinical trial. Journal of Oral Implantology. 2015; 41: 281-286.

8. Trisi, P., Berardini, M., Falco, A. and Podaliri Vulpiani, M. Effect of implant thread geometry on secondary stability, bone density and bone-to-implant contact: a biomechanical and histological analysis. Implant Dentistry. 2015; 24: 384-391.

9. Shibata, Y. and Tanimoto, Y. A review of improved fixation methods for dental implants. Part I: surface optimization for rapid osseointegration. Journal of Prosthodontic Research. 2015; 59: 20-33.

10. Trisi P, Berardini M, Falco A and Vulpiani MP. Validation of value of actual micromotion as a direct measure of implant micromobility after healing (secondary implant stability). An in vivo histologic and biomechanical study Clin. Oral Impl. Res. 2016; 27: 1423-1430.

11. Freitas AC Jr, Bonfante EA, Giro G, Janal MN, Coelho PG. The effect of implant design on insertion torque and immediate micromotion. Clin Oral Implants Res. 2012; 23:113-118.

12. Rabel A, Köhler SG, Schmidt-WesthausenAM. Clinical study on the primary stability of two dental implant systems with resonance frequency analysis. Clin Oral Investig. 2007; 11: 257-265.

13. Premnath K, Sridevi J, Kalavathy N, Nagaranjani P, Sharmila MR . Evaluation of stress distribution in bone of different densities using different implant designs: a threedimensional finite element analysis. J Indian Prosthodont Soc 2013; 13: 555-559.

14. Kim DS, Lee WJ, Choi SC, Lee SS, Heo MS, Huh KH, Kim TI and Yi WJ. Comparison of dental implant stabilities by impact response and resonance frequencies using artificial bone. Med Eng Phys. 2014; 36:715-720.

15. Mazzo CR, Reis AC, Shimano AC and Valente ML. In vitro analysis of the influence of surface treatment of dental implants on primary stability. Braz Oral Res. 2012; 26:313-317.

16. Jung UW, Kim S, Lee IK, Kim MS, Lee JS and Kim HJ. Secondary stability of microthickness hidroxyapatite-coated dental implants installed without primary stability in dogs. Clin Oral Implants Res. 2013; 25:1169-1174.

17. Lopez de Oliveira G, Leite F, Pontes A, Sakakura C, Junior E. Comparison of and secondary stability of implants with anodized surfaces and implants treated by acids: a splitmouth randomized controlled clinical trial. J Oral Maxillofac Implant. 2016; 31: 186-190.

18. Abuhussein H, Pagni G, Rebaudi A, Wang HL. The effect of thread pattern upon implant osseointegration: review. Clin Oral Implants Res. 2010; 21: 129-136. 
19. Szmukler-Moncler S, Salama H, Reingewirtz Y, Dubruille JH. Timing of loading and effect of micromotion on bonedental implant interface: review of experimental literature. J BiomedMater Res. 1998; 43: 192-203.

20. Dos Santos MV, Elias CN, Cavalcanti Lima JH. The effects of superficial roughness and design on the primary stability of dental implants. Clin Implant Dent Relat Res. 2011; 13: 215-223

21. Pozzi A, Tallarico M, Moy PK. Immediate loading with a novel implant featured by variable-threaded geometry, internal conical connection and platform shifting: three-year results from a prospective cohort study. Eur J Oral Implant. 2015; 8: 51-63.

22. Pozzi A, Mura P. Immediate loading of conical connection implants: up-to-2-year retrospective clinical and radiologic study. Int J Oral Maxillofac Implants. 2016;31:142-152.

23. Schiefer H, Bram M, Buchkremer HP, Stöver D. Mechanical examinations on dental implants with porous titanium coating. J Mater Sci Mater Med 2009;20:1763-1770.

24. Abu-Hammad O, Khraisat A, Dar-Odeh N, El-Maaytah M. Effect of dental implant cross-sectional design on cortical bone structure using finite element analysis. Clin Implant Dent Relat Res 2007; 9: 217-221.

25. Watari F, Yokoyama A, Saso F, Uo M, Kawasaki T. Fabrication and properties of functionally graded dental implant. Compos Part BEng 1997; 28: 5-11.

26. Waechter J, Madruga M, Carmo Filho LC, Manzolli Leite FR, Schinestsck AR, Fernanda Faot F. Comparison between tapered and cylindrical implants in the posterior regions of the mandible: A prospective, randomized, split-mouth clinical trial focusing on implant stability changes during early healing Clin Implant Dent Relat Res. 2017;19:733-741

27. Unger AS, Lewis RJ, Gruen T. Evaluation of a porous tantalum uncemented acetabular cup in revision total hip arthroplasty: Clinical and radiological results of 60 hips. J Arthroplasty 2005; 20: 1002-1009.

28. Bencharit S, Byrd WC, Altarawneh S, et al. Development and applications of porous tantalum trabecular metal-enhanced titanium dental implants. Clin Implant Dent Relat Res 2013; 3: 559-567.

29. Implantology Catalog TiXos line (C) 2013 Leader Italia srl

30. Diana-Irinel Băilă. Dental Restorations of Co-Cr Using Direct Metal Laser Sintering Process International Journal of Materials, Mechanics and Manufacturing. 2018; 6(2): 94-98.

31. Niinomi M. Mechanical biocompatibilities of titanium alloys for biomedical applications. J Mech Behav Biomed Mater 2008; 1: 30-42.

32. Guillemot F. Recent advances in the design of titanium alloys for orthopedic applications. Expert Rev Med Devices 2005; 2: 741-748.

33. Bhattarai SR, Khalil KA, Dewidar M, Hwang PH, Yi HK and Kim HY. Novel production method and in-vitro cell compatibility of porous Ti-6Al-4V alloy disk for hard tissue engineering. J Biomed Mater Res. 2008; 86: 289-299.

34. Head WC, Bauk DJ, Emerson RH. Titanium as the material of choice for cementless femoral components in total hip arthroplasty. Clin Orthop Relat Res 1995; 311: 85-90.

35. Oh IH, Nomura N, Masahashi N, Hanada S. Mechanical properties of porous titanium compacts prepared by powder sintering. Scripta Mater 2003; 49: 1197-1202.

36. Schiefer H, Bram M, Buchkremer HP and Stöver D. Mechanical examinations on dental implants with porous titanium coating. J Mater Sci Mater Med 2009;20:1763-1770.

37. Bayarchimeg D, Namgoong H, Kim BK, Kim MD, Kim S, Kim TI, Seol YJ, Lee YM, Ku Y, Rhyu IC, Lee EH, Koo $\mathrm{KT}$. Evaluation of the correlation between insertion torque and primary stability of dental implants using a block bone test. J Periodontal Implant Sci 2013; 43: 30-36

38. Romanos GE, Ciornei G, Malmstrom H and Gupta B. In Vitro Assessment of Primary Stability of Straumann ${ }^{\circledR}$ Implant Designs Clin Imp Dent and Related Res. 2014; 16(1): $89-95$

39. Chowdhary R, Jimbo R, Thomsen C, Carlsson L, Wennerberg A. Biomechanical evaluation of macro and micro designed screw-type implants: an insertion torque and removal torque study in rabbits. Clin Oral Implants Res. $2013 ; 24: 342-346$

40. Costa Valente ML, Castro DT, Shimano AC, Lepri CP and Reis AC. Analysis of the influence of implant shape on primary stability using the correlation of multiple methods. Clin Oral Invest 2015; 19:1861-1866

41. Nkenke E, Kloss F, Wiltfang J, et al. Histomorphometric and fluorescence microscopic analysis of bone remodeling after installation of implants using an osteotome technique. Clin Oral Impl Res 2002; 13:595-602. 
42. Beuchter A, Kleinheinz J, Wiesmann HP, et al. Biological and biomechanical evaluation of bone remodelling and implant stability after using an osteotome technique. Clin Oral Implants Res 2005; 16:1-8.

43. Buser D, Broggini N, Wieland M, et al. Steinemann. Enhanced bone apposition to a chemically modified SLA titanium surface. J Dent Res. 2004; 83:529-533.

44. McCullough JJ, Klokkevold PR. The effect of implant macro-thread design on implant stability in the early postoperative period: a randomized, controlled pilot study. Clin Oral Implants Res. 2016; 211-245.

45. Trisi P, Berardini M, Falco A, Podaliri Vulpiani M. Validation of value of actual micromotion as a direct measure of implant micromobility after healing (secondary implant stability). An in vivo histologic and biomechanical study. Clin Oral Implants Res 2016;27: 1423-1430.

46. Hsiang-Hsi Hong, Adrienne Hong, Lan-Yan Yang, WeiYang Chang,Yi-Fang Huang and Yen-Ting Lin. Implant Stability Quotients of Osteotome Bone Expansion and Conventional Drilling Technique for $4.1 \mathrm{~mm}$ Diameter Implant at Posterior Mandible Clinical Implant Dentistry and Related Research. 2017; 19(2): 253-260.

47. Deporter DA, Watson PA, Pilliar RM, et al. A histological assessment of the initial healing response adjacent to porous-surfaced, titanium alloy dental implants in dogs. J Dent Res 1986; 65: 1064-1070. 